\title{
The Structure of Galactic Gas at High Latitudes: the Southern Polar Cap
}

\author{
I. V. Gosachinskij", G. N. Il'in, and V. A. Prozorov \\ Special Astrophysical Observatory, St. Petersburg Branch, Russian Academy of Sciences, Pulkovo, St. \\ Petersburg, 196140 Russia \\ Received July 30, 2003
}

\begin{abstract}
We analyze the angular structure of the $21-\mathrm{cm}$ interstellar neutral hydrogen emission at six and seven declinations in the northern (published previously) and southern polar caps of the Galaxy (Galactic latitudes from $-40^{\circ}$ to $-90^{\circ}$ ), respectively, with an extent of $90^{\circ}$ in right ascension. The RATAN-600 radio telescope has a beam width averaged over these regions of $2^{\prime} .0 \times 30^{\prime}$. One-dimensional power spectra for the angular distribution of interstellar neutral hydrogen emission were computed in each $6.3-\mathrm{km} \mathrm{s}^{-1}$-wide spectral channel by using the standard fast Fourier transform (FFT) code and were smoothed over $1^{\mathrm{h}}$ in right ascension. The Galactic latitude dependence of the mean parameters for the sky distribution of $\mathrm{HI}$ line emission at high latitudes was found to correspond to the distribution of gas in the form of a flat layer only in the northern region, while in the southern cap, the gas distribution is much less regular. In addition, the mean $\mathrm{H}$ I radial velocities are negative everywhere $\left(-3.7 \pm 3.0 \mathrm{~km} \mathrm{~s}^{-1}\right.$ in the north and $-6.0 \pm 2.4 \mathrm{~km} \mathrm{~s}^{-1}$ in the south). The power spectra of the angular fluctuations in the range of angular periods from $10^{\prime}$ to $6^{\circ}$ appear as power laws. However, the spectral indices change greatly over the sky: from -3 to -1.2 ; on average, as the Galactic latitude increases and the $\mathrm{H} \mathrm{I}$ column density decreases, the fluctuation spectrum of the interstellar gas emission becomes flatter. In the northern polar region, this behavior is much more pronounced, which probably stems from the fact that the gas column density in the south is generally a factor of 2 or 3 higher than that in the north. Therefore, the spectra are, on average, also steeper in the south, but the dependence on Galactic latitude is weaker. Using simulations, we show that the observed power-law spectrum of the $\mathrm{HI}$ emission distribution can be obtained in terms of not only a turbulent, but also a cloud model of interstellar gas if we use our previous spectra of the diameters and masses of $\mathrm{H} \mathrm{I}$ clouds. (C) 2004 MAIK “Nauka/Interperiodica”.
\end{abstract}

Key words: interstellar medium, neutral hydrogen, cloud structure.

\section{INTRODUCTION}

The characteristics of the $\mathrm{H}$ I cloud structure determined from observations play a major role in elucidating the main physical processes that govern its existence: heating and cooling, cloud-cloud collisions, molecular cloud formation, gravitational instability and star formation, etc.

There are many methods for studying the structure of the interstellar medium. The natural view of a cloud as an object that is separated from an extended background and neighboring objects by the intensity distribution of its emission is taken in many observational works. However, several authors (Crovisier and Dickey 1983; Green 1993; Dickey et al. 2001) used a statistical method: they measured the spatial spectrum of the $\mathrm{H} \mathrm{I}$ intensity distribution with interferometers or single dishes. The major advantage of the spectral approach is its high sensitivity to the angular characteristics of the gas emission, but only

\footnotetext{
E-mail: gos@fsao.spb.su
}

if these characteristics are stationary in regions of significant angular sizes. It should also be noted that, first, the measured spectra are angular rather than spatial, second, all authors measure the so-called power spectra, i.e., the spectra of the squares of the amplitudes with the loss of phase information, and, third, small areas in the Galactic plane where the distribution of interstellar gas emission is far from stationary and strongly depends on the radial velocity are chosen for measurements.

The angular spectra obtained by the above authors are close to power laws. However, as Green (1993) pointed out, the spectral indices measured with interferometers (two-dimensional spectra) differ by unity from the one-dimensional spectra computed by using the Fourier transform of the drift curves obtained with single dishes. The two-dimensional spectra are steeper, and their amplitudes are smaller.

The papers by Lazarian (1995), Lazarian and Pogosyan (2000), and Goldman (2000) are also noteworthy. These authors made attempts to mathematically interpret the observed angular spectra in 
an effort to directly calculate the three-dimensional statistics of interstellar gas fluctuations and its relationship to the turbulence characteristics of the interstellar medium, which is believed to determine these observed spectra.

Taking into account all these circumstances, in 1996 we embarked on a program of measuring the angular spectra of the $21-\mathrm{cm} \mathrm{H} \mathrm{I} \mathrm{emission} \mathrm{at}$ high Galactic latitudes with the RATAN-600 radio telescope. Previously (Gosachinskij et al. 1999), we published the results of our study of the northern Galactic polar cap. Here, we present the results of our observations of a region around the southern Galactic pole and make a comparison of these data. Note that the gas located near the Sun (300-600 pc) at nearly zero radial velocities $\left( \pm 20 \mathrm{~km} \mathrm{~s}^{-1}\right)$ is mainly observed at high Galactic latitudes. In contrast to the angular distribution of its emission near the Galactic plane, its observed angular characteristics are expected to be much more homogeneous statistically in this case. Therefore, it is hoped that the application of spectral methods for studying the structural properties of the distribution of interstellar gas emission averaged over the sky on a large scale is adequate.

\section{INSTRUMENTATION AND TECHNIQUES}

The RATAN-600 antenna has a $2.0 \times 30^{\prime}$ fieldaveraged beam FWHM and an effective area of 900$1000 \mathrm{~m}^{2}$ at a wavelength of $21 \mathrm{~cm}$ at declinations from $-40^{\circ}$ to $+10^{\circ}$. The automated radiospectrometric system of the radio telescope has an uncooled HEMT amplifier at its input at decimeter wavelengths (Il'in et al. 1997). In our observations, we used a 39channel filter spectrum analyzer (Venger et al. 1982) with a channel bandwidth of $30 \mathrm{kHz}\left(6.3 \mathrm{~km} \mathrm{~s}^{-1}\right)$ and a separation between the channels of $30 \mathrm{kHz}$, so the full bandwidth of the survey was $1.2 \mathrm{MHz}$. The time constant of the output devices was $6 \mathrm{~s}$, and the sampling period was $3 \mathrm{~s}$. An IBM PC was used for system control and data acquisition and primary reduction through KAMAK (Alferova et al. 1986; Venger et al. 1997). Observations of a series of bright references sources with measured fluxes (Venger et al. 1981) were used to check the parameters of the antenna and the instrumentation.

The southern polar region was observed during 1999-2003. Drift curves were obtained at declinations of $-40^{\circ},-34^{\circ},-27^{\circ},-20^{\circ},-10^{\circ}, 0^{\circ}$, and $+10^{\circ}$ in the range of right ascensions from $22^{\mathrm{h}}$ to $4^{\mathrm{h}}$ (Galactic latitudes from $-40^{\circ}$ to $-90^{\circ}$ ). At each declination, we carried out two sets of observations, each containing 10 to 14 records, with a spectrum analyzer frequency shift by half the separation between the spectral channels, so each drift curve ultimately contained 78 spectral channels that followed at 3.15$\mathrm{km} \mathrm{s}^{-1}$ steps. The mean square of the noise fluctuations in the averaged records was $0.1 \mathrm{~K}$. We measured all radial velocities relative to the Local Standard of Rest. In addition, we obtained one more drift curve for the northern polar cap at a declination of $-5^{\circ}$; the results of its processing are presented below in figures.

At high latitudes, where the total intensity of the $21-\mathrm{cm} \mathrm{H}$ I emission is low, the radio line profiles are known to consist of the following three main components: the narrow emission line near zero radial velocities that originates in an $\mathrm{H} I$ layer near the Sun, the emission from rare $\mathrm{H}$ I clouds at high negative and positive radial velocities, and the stray radiation from the far sidelobes and diffuse background of the antenna system. An example of a profile that clearly shows the first and the third components is given in our previous paper (Gosachinskij et al. 1999). Eliminating the stray diffuse background is a serious problem, and special methods were developed for its solution (see, e.g., Heiles et al. 1981; McGee and Newton 1986; Hartmann et al. 1996). However, as we showed previously, this radiation may be ignored in the formulated problem, because the pattern of the diffuse background of the antenna cannot give smallscale features in the intensity distribution. In general, the large-scale $\mathrm{H}$ I distribution at high latitudes was studied in pioneering works (see, e.g., Fejes and Wesselius (1973) and references therein). Our data on the total gas column density that we obtained after eliminating the stray diffuse background smoothed by the RATAN-600 beam are used only to compare the total H I column density with the fine-structure parameters determined with the same instrument using one coordinate.

We computed the one-dimensional angular spectrum of the interstellar gas intensity distribution in each spectral channel by using the standard FFT code based on 64 points. Consequently, for a sampling period of $3^{\mathrm{s}}$, the first and the last (32nd) spectral harmonics correspond to periods in right ascension of $192^{\mathrm{s}}$ and $6^{\mathrm{s}}$, respectively. At $\delta=0^{\circ}$, these periods correspond to angular periods of $48^{\prime}$ and $1^{\prime} .5$, respectively. At other declinations, these periods increase in inverse proportion to $\cos \delta$. It emerged that the sensitivity of the instrumentation achieved in our survey allowed a signal to be isolated at spatial harmonics with numbers no higher than 8 to 10 . Therefore, we subsequently smoothed the drift curves by the function $\sin x / x$ with a sine period of $24^{\mathrm{s}}$ so as to be able to compute the spectra for periods from $48^{\mathrm{s}}$ to $1536^{\mathrm{s}}$ using the same FFT code based on 64 points with appropriate resampling while avoiding the so-called aliasing effect. 
Table 1. Mean radial velocities of $\mathrm{H}$ I at low radial velocities in the southern polar cap

\begin{tabular}{c|c|c|c|c|c|c|c|c|c|c|c}
\hline & \multicolumn{7}{|c|}{$\alpha$} \\
\hline$\delta$ & $03^{\mathrm{h}} 30^{\mathrm{m}}$ & 0300 & 0230 & 0200 & 0130 & 0100 & 0030 & 0000 & 2330 & 2300 & 2230 \\
\hline+10.0 & -2.3 & -5.3 & -4.5 & -8.5 & -8.3 & -6.8 & -6.8 & -6.8 & -6.8 & -4.5 & -2.0 \\
& +9.0 & +10.5 & +12.8 & +13 & +13 & +13 & +12.8 & +12.8 & +12.8 & +12.0 & +10.5 \\
0.0 & -3.0 & -5.0 & -7.0 & -8.0 & -8.0 & -8.0 & -6.0 & -2.0 & -1.5 & -2.0 & -0.5 \\
& +8.0 & +8.0 & +7.5 & & & & & & & & \\
-10.0 & -4.7 & -4.7 & -7.5 & -10.3 & -8.4 & -8.4 & -8.4 & -5.6 & -2.8 & -0.9 & -0.9 \\
& +9.3 & +8.4 & +7.5 & +9.3 & & & & & & & \\
-20.0 & -6.6 & -10.3 & -11.3 & -9.4 & -8.4 & -10.3 & -9.4 & -4.7 & -3.8 & -3.8 & -4.7 \\
& +7.5 & +1.5 & 0.0 & 0.0 & & & & & & & \\
-27.0 & -3.7 & -8.4 & -7.5 & -8.4 & -7.5 & -5.6 & -5.6 & -3.7 & -3.7 & -5.6 & -5.6 \\
-34.0 & -3.7 & -4.7 & -6.0 & -6.5 & -6.5 & -6.9 & -7.3 & -5.6 & -5.6 & -5.6 & -7.1 \\
-40.0 & -5.6 & -6.2 & -7.5 & -8.4 & -8.4 & -8.4 & -6.5 & -6.5 & -5.6 & -5.6 & -3.7 \\
& -28 & & & & & & & & & & \\
\hline
\end{tabular}

The spectra of the squares of the amplitudes (i.e., the spatial power spectra) were smoothed over a $1^{\mathrm{h}}\left(15^{\circ}\right)$ interval and along the radial velocity axis over five spectral channels $\left(15.7 \mathrm{~km} \mathrm{~s}^{-1}\right)$. Subsequently, the system noise spectrum obtained as a spectrum averaged over the spectrometer channels where no line emission was observed was subtracted from the maximum spectrum. Since the system noise does not correlate with the fluctuations in the $\mathrm{H} I$ line intensity distribution recorded by the RATAN-600 antenna, their spectra are added quadratically (in power). This is how they should be separated, and therein lies the physical meaning of the angular power spectrum. Subsequently, we corrected the difference spectrum for the following three effects that smoothed the observed fluctuations: smoothing by the horizontal antenna beam, filtering by the output low-frequency devices of the spectrometer, and averaging of the fluctuations over the vertical antenna beam.

Thus, we obtained 11 statistically significant spectra computed at 0.5 steps in each drift curve with an extent of $6^{\mathrm{h}}$ in right ascension. The fluctuation amplitude of the interstellar gas emission at very low angular frequencies is known to be large. Since, on the one hand, angular periods no larger than a few degrees were of special interest, and, on the other hand, there is always the risk of spurious signals leaking through the sidelobes of the transfer function of the FFT spectrum analyzer in a spectral analysis, we removed the periods longer than $6^{\circ}$ as well as the constant component from the drift curves in all channels.

\section{RESULTS \\ The Total H I Column Density}

The radial velocities of the $\mathrm{H}$ I peaks in the region around the southern Galactic pole under study are listed in Table 1. Since no data of this kind were published in our previous paper for the northern region, we give them here in Table 2 to conveniently compare the data on both regions. Our data confirm the long-known fact that the mean radial velocities of the circumsolar gas are mainly negative both in the south $\left(-6.0 \pm 2.4 \mathrm{~km} \mathrm{~s}^{-1}\right)$ and in the north $(-3.7 \pm$ $3 \mathrm{~km} \mathrm{~s}^{-1}$ ). No clear systematic correlation of the H I radial velocity with coordinate (e.g., Galactic latitude, Fig. 1) is observed both in the north and in the south. A slow fall of the background gas in the layer toward its middle plain may serve as the only plausible explanation of this phenomenon. This assumption also accounts for the choice of the $x$ axis in Fig. 1: if the spatial velocity is mainly perpendicular to the Galactic plane, then the radial velocity is proportional to $V_{\mathrm{g}} \sin (b)$. 
Table 2. Mean radial velocities of $\mathrm{H}$ I at low radial velocities in the northern polar cap

\begin{tabular}{c|c|r|r|r|r|r|r|r|r|r|c}
\hline & \multicolumn{10}{c}{$\alpha$} \\
\hline \multicolumn{1}{c|}{} & \multicolumn{1}{c|}{$5^{\mathrm{h}} 30^{\mathrm{m}}$} & 1500 & 1430 & 1400 & 1330 & 1300 & 1230 & 1200 & 1130 & 1100 & 1030 \\
\hline+50.0 & -5.0 & -4.0 & -3.0 & -5.0 & -7.0 & -8.0 & -7.0 & -7.0 & -9.0 & -12.0 & -14.0 \\
+39.0 & -1.5 & -1.5 & -2.0 & 0.0 & 0.0 & -2.0 & -2.5 & - & - & - & - \\
+27.5 & -3.0 & -1.5 & -1.5 & -3.5 & -5.0 & - & - & - & - & -7.0 & 0.0 \\
+16.0 & -0.5 & -0.5 & 0.0 & +0.5 & 0.0 & 0.0 & +0.5 & 0.0 & -1.0 & -5.0 & -5.5 \\
+5.0 & -2.5 & -2.5 & -2.5 & -2.5 & -3.0 & -3.5 & -5.0 & -5.0 & -6.0 & -7.0 & -5.04 \\
-5.0 & 0.0 & 0.0 & 0.0 & 0.0 & -6.0 & -6.0 & -6.0 & -6.0 & -8.3 & -7.5 & -6.0 \\
& & & & & +3.0 & +2.3 & +3.0 & +3.0 & +7.5 & +2.3 & +3.3 \\
\hline
\end{tabular}

Some of the areas in the regions under study (mainly at low latitudes) are characterized by profile splitting. In this case, we gave the radial velocities of both peaks in Tables 1 and 2. The appearance of $\mathrm{H} \mathrm{I}$ emission at positive velocities can be easily explained. The point is that a large-scale H I structure at nearly zero radial velocities that is commonly called ridges is known to exist at high latitudes. Fejes and Wesselius (1973) gave parameters for 13 such ridges; most of them (11) are almost perpendicular to the Galactic plane, and some of them reach latitudes of $\pm 40^{\circ}$. Note also that, in contrast to the background gas, many of them have positive radial velocities, and five ridges fall within the region of our survey precisely where the peaks at positive velocities are observed. Since any large-scale structure is not the goal of our study, these peaks are not considered below. The FWHMs of the H I profile peaks corrected for the analyzer band smoothing effect are shown in Fig. 2 for the northern and southern polar caps. The mean FWHMs are $13.4 \pm 3.2 \mathrm{~km} \mathrm{~s}^{-1}$ in the south and $9.8 \pm 3.0 \mathrm{~km} \mathrm{~s}^{-1}$ in the north; there is no correlation with coordinates. Finally, the H I column density in the southern polar region is given in Table 3 , and its plot against cosec $|b|$ for the north and the south is shown in Fig. 3. Since the abscissas in Figs. 2 and 3 are proportional to the extent of the line of sight for an observer within the flat layer, it is interesting to note that the layer appears flat only in the northern part. In the south, the gas column density, first, a factor of 2 or 3 higher than that in the north, and, second, its distribution is much less regular. Our conclusions are consistent with the data by Fejes and Wesselius (1973) and with the fact that the inclined H I disk discovered by these authors does not fall within the region of our survey, because it lies at lower latitudes.

\section{Angular Spectra of the H I Line Emission}

In general, the one-dimensional angular fluctuation power spectra of the $21-\mathrm{cm} \mathrm{H}$ I line intensity distribution in the southern Galactic polar cap also appear as power laws as those in the northern region (Gosachinskij et al. 1999). The spectral indices, which are the slopes of the regression line, are given together with their rms computational errors in Table 4 . As in the north, the angular spectra are characterized by a large spread in indices: from -1.2 to -3 . Although the relation between the angular scale of the spectra and the time scale depends on declination, it is clear that this merely displaces the angular spectra on a logarithmic scale along the $x$ axis without changing their slopes (spectral indices).

The spectral indices for the northern and southern regions can be compared with one another and with other parameters by using Figs. 4 and 5 . Since we do not know how the spectral index can depend on latitude, we took the latitude in degrees as the abscissa in Fig. 4. The northern and southern polar caps differ not only in total gas column density (Fig. 3), but also in gas structure. In the southern region, the spectral index correlates much better with Galactic latitude than it does in the northern region (Fig. 4, the correlation coefficient is -0.7 ), although, in general, the fluctuation spectra become flatter with increasing Galactic latitude in both regions.

Surprisingly, the clear correlation between spectral index and total gas column density that we found previously for the northern region (Fig. 5) is much less distinct in the southern polar cap. However, it 


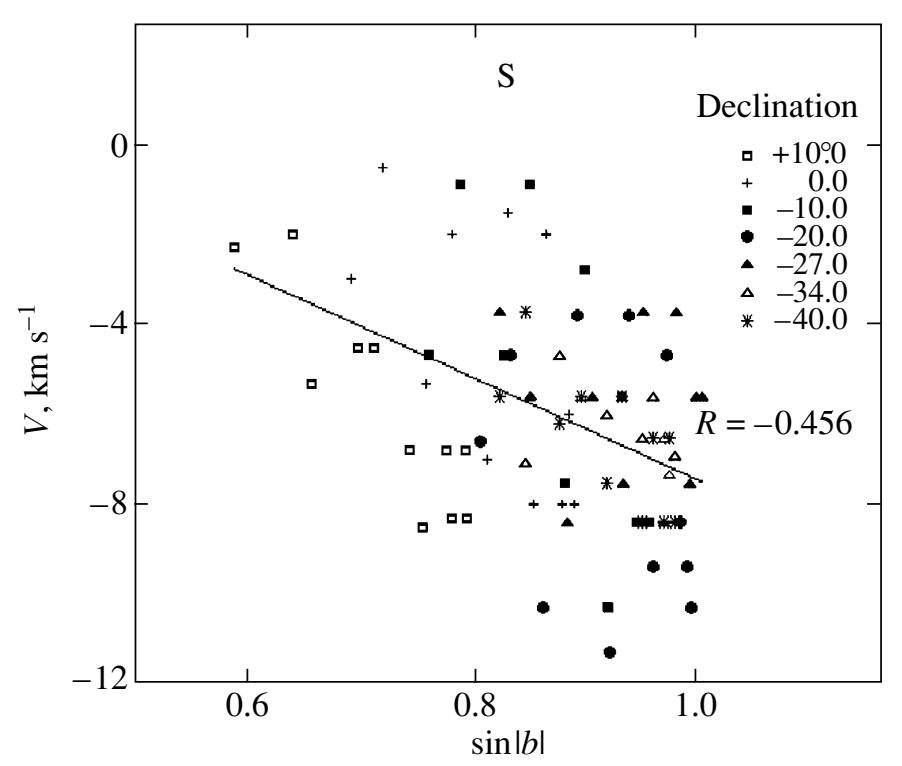

Fig. 1. H I radial velocity versus Galactic latitude in the southern polar cap. The straight line in this and the following figures represents a linear regression, and $R$ is the correlation coefficient between the quantities in the plots.
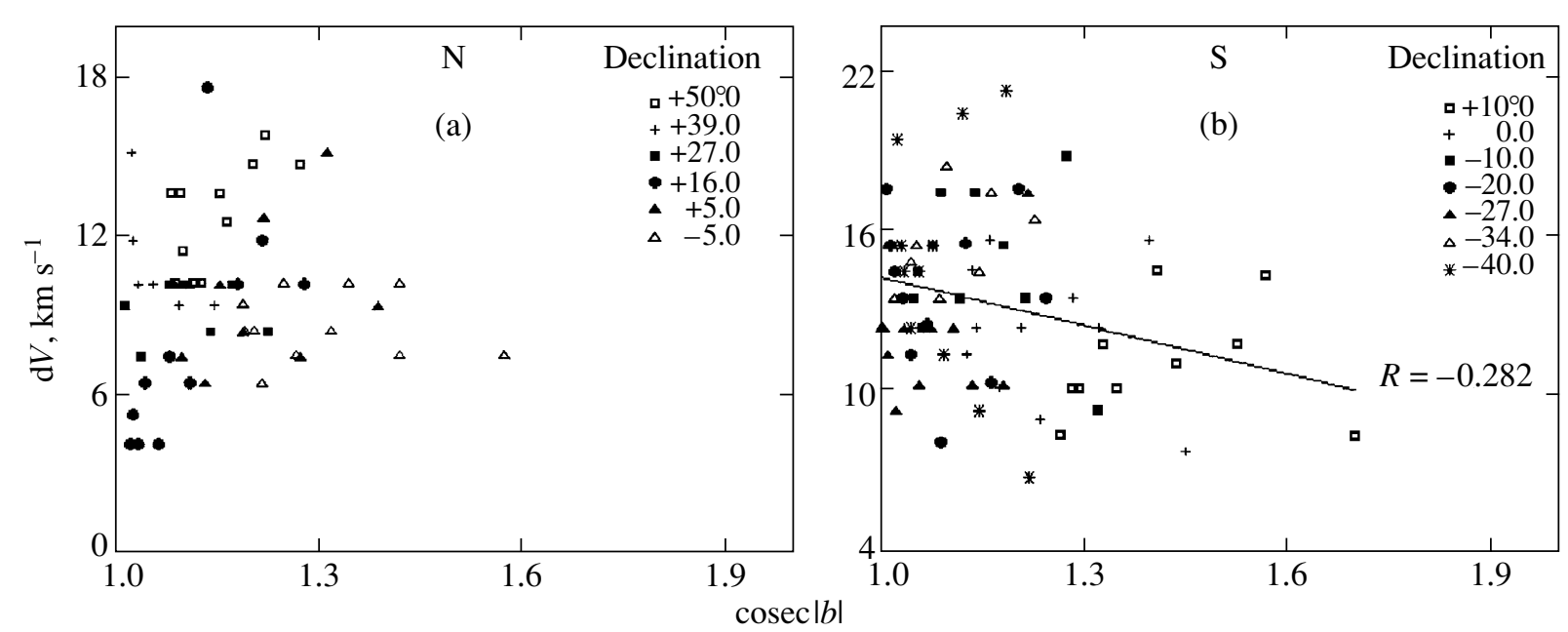

Fig. 2. H I profile FWHMs at low radial velocities versus cosec $|b|$ in the northern (a) and southern (b) polar caps.

should be borne in mind that the detected flattening of the fluctuation spectra can have a different nature: this can be the physical effect that stems from the fact that features of small angular sizes at a high total density do not survive through collisions or evaporation in the hot intercloud medium, causing the observed angular spectra to become steeper; on the other hand, a selection effect can also act-when the number of small features is large, they are difficult to discern because of the insufficient sensitivity and angular resolution, which cannot be offset by correcting the spectra for smoothing effects.

\section{DISCUSSION}

Two distinctly different physical processes are usually invoked to explain the physical nature of the cloud structure of the interstellar medium. One of them, interstellar turbulence, is basically statistical, i.e., the characteristic interstellar gas velocity field leads to turbulent fluctuations in gas density-to what observers commonly call clouds. A description of this physics can be found in many papers, for example, in the fundamental paper by BallesterosParedes et al. (1999), which contains a large number of references to other studies.

This approach, which may be attractive mathematically, is often unacceptable for observers for 
Table 3. Mean H I column densities (in units of $10^{20} \mathrm{~cm}^{-2}$ ) in the southern polar cap

\begin{tabular}{c|c|c|c|c|c|c|c|c|c|c|c}
\hline & \multicolumn{10}{c}{$\alpha$} \\
\hline$\delta$ & $03^{\mathrm{h}} 30^{\mathrm{m}}$ & 0300 & 0230 & 0200 & 0130 & 0100 & 0030 & 0000 & 2330 & 2300 & 2230 \\
\hline+10.0 & 2.60 & 3.50 & 5.00 & 4.40 & 3.80 & 3.40 & 3.80 & 3.90 & 3.00 & 2.90 & 3.6 \\
& 9.00 & 6.40 & 1.30 & 0.50 & 0.30 & 0.40 & 0.50 & 0.20 & 0.20 & 0.20 & 0.50 \\
0.0 & 1.00 & 1.30 & 1.00 & 1.10 & 1.30 & 1.30 & 2.00 & 3.50 & 3.50 & 4.40 & 4.50 \\
& 5.60 & 2.70 & 1.30 & & & & & & & & \\
-10.0 & 0.75 & 1.50 & 1.60 & 1.90 & 1.80 & 2.20 & 2.40 & 2.30 & 1.90 & 2.50 & 3.20 \\
& 3.10 & 1.60 & 0.23 & 0.02 & & & & & & & \\
-20.0 & 0.99 & 1.30 & 0.87 & 0.53 & 0.81 & 1.10 & 0.87 & 1.20 & 1.40 & 1.50 & 1.60 \\
& 1.30 & 0.70 & 0.80 & 0.45 & & & & & & & \\
-27.0 & 0.56 & 0.59 & 0.58 & 0.52 & 0.39 & 0.58 & 0.94 & 0.71 & 0.77 & 0.79 & 0.68 \\
-34.0 & 0.59 & 1.15 & 1.33 & 1.23 & 1.24 & 1.49 & 0.83 & 0.66 & 0.44 & 0.65 & 0.43 \\
-40.0 & 0.17 & 0.59 & 0.78 & 1.20 & 1.50 & 2.50 & 1.70 & 1.40 & 1.60 & 1.90 & 1.30 \\
\hline
\end{tabular}

Table 4. Indices of the angular H I emission spectrum at low negative radial velocities in the southern polar cap

\begin{tabular}{c|r|r|r|r|r|r|r|r|r|r|r}
\hline & \multicolumn{10}{c}{$\alpha$} \\
\hline$\delta$ & \multicolumn{1}{|c|}{$03^{\mathrm{h}} 30^{\mathrm{m}}$} & 0300 & 0230 & 0200 & 0130 & 0100 & 0030 & 0000 & 2330 & 2300 & 2230 \\
\hline+10.0 & -3.3 & -2.8 & -3.0 & -2.9 & -2.7 & -2.9 & -2.9 & -2.5 & -2.5 & -2.9 & -3.0 \\
& 0.1 & 0.2 & 0.2 & 0.2 & 0.3 & 0.2 & 0.2 & 0.2 & 0.2 & 0.2 & 0.2 \\
0.0 & -2.8 & -2.6 & -2.3 & -2.4 & -2.3 & -2.6 & -2.5 & -2.6 & -2.6 & -2.5 & -2.7 \\
& 0.1 & 0.1 & 0.3 & 0.2 & 0.3 & 0.2 & 0.3 & 0.2 & 0.2 & 0.2 & 0.2 \\
-10.0 & -2.8 & -3.0 & -2.1 & -2.4 & -2.2 & -2.3 & -2.0 & -2.0 & -2.1 & -2.6 & -2.8 \\
& 0.1 & 0.1 & 0.2 & 0.2 & 0.2 & 0.2 & 0.2 & 0.2 & 0.2 & 0.1 & 0.1 \\
-20.0 & -3.0 & -2.5 & -1.7 & -2.7 & -2.2 & -2.1 & -1.9 & -1.8 & -2.3 & -2.4 & -2.6 \\
& 0.2 & 0.2 & 0.3 & 0.4 & 0.3 & 0.2 & 0.3 & 0.3 & 0.2 & 0.3 & 0.3 \\
-27.0 & -1.9 & -1.9 & -2.2 & -2.3 & -2.5 & -1.8 & -2.7 & -2.0 & -2.1 & -2.2 & -2.2 \\
& 0.3 & 0.2 & 0.3 & 0.3 & 0.3 & 0.3 & 0.5 & 0.3 & 0.2 & 0.3 & 0.3 \\
-34.0 & -2.7 & -2.4 & -2.5 & -2.3 & -1.2 & -1.9 & -1.5 & -2.4 & -3.0 & -2.7 & -2.3 \\
& 0.3 & 0.4 & 0.2 & 0.3 & 0.4 & 0.3 & 0.2 & 0.3 & 0.2 & 0.5 & 0.4 \\
-40.0 & -2.7 & -2.4 & -2.5 & -2.3 & -1.2 & -1.9 & -1.5 & -2.4 & -3.0 & -2.7 & -2.2 \\
& 0.3 & 0.4 & 0.2 & 0.3 & 0.4 & 0.3 & 0.2 & 0.3 & 0.2 & 0.5 & 0.4 \\
\hline
\end{tabular}

the following simple reason: the cloud structure that arises from turbulence must be hierarchical in principle; i.e., clouds of smaller angular sizes must be within clouds of larger sizes. Unfortunately, there are virtually no observational works that would unambiguously reveal such a structure. Clouds of interstellar gas most commonly appear as separate inde- pendent objects. In addition, in our case, the problem is complicated by the fact that the observed region cannot be considered to be a thin layer, so the results of theoretical model calculations such as those by Lazarian (1995) or Goldman (2000) are inapplicable.

The long-existing two-stage scenario in which clouds originate from a continuous medium through 


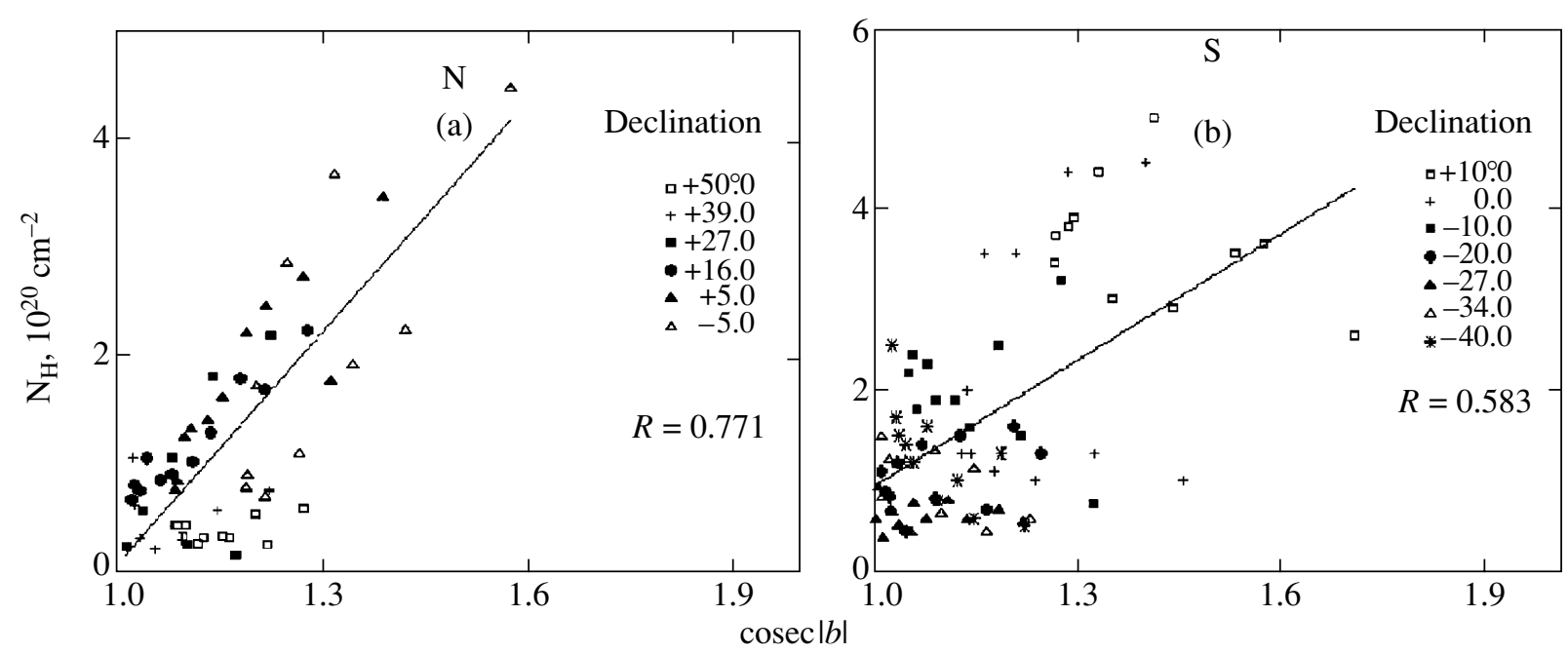

Fig. 3. H I column densities in the northern (a) and southern (b) polar caps.

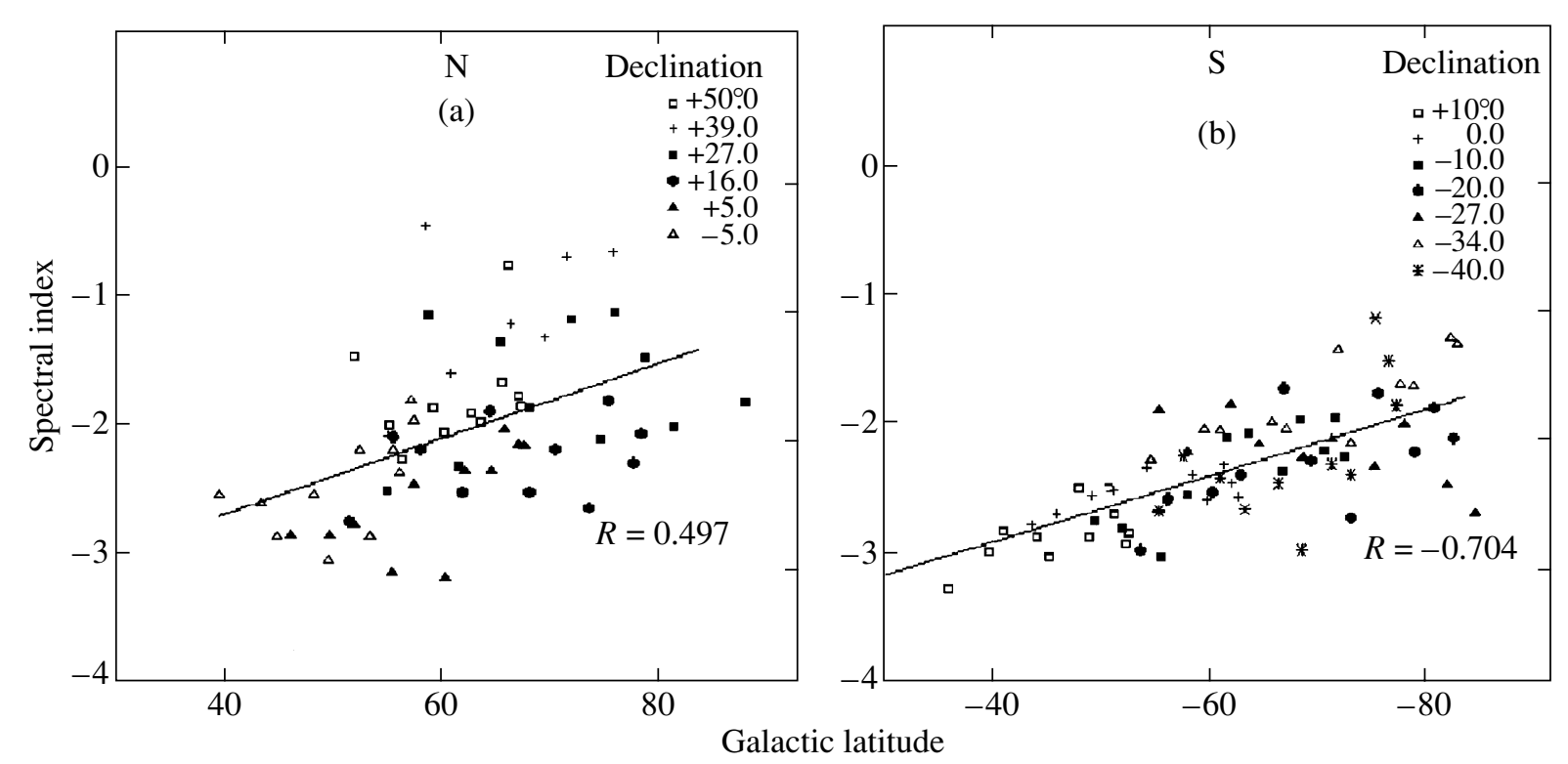

Fig. 4. Indices of the one-dimensional angular H I fluctuation spectra in the northern (a) and southern (b) Galactic polar caps versus Galactic latitude.

any of the instabilities, most commonly the thermal instability that arises after initial contraction of the medium in shocks - spiral arms or supernova remnants, is taken as an alternative. Subsequently, the clouds exist in equilibrium with the external intercloud gas pressure, inelastically colliding between themselves. As a result of these collisions, they increase in mass and density until gas molecularization begins and Jeans instability starts working. This scenario has been considered by many authors, so we refer the reader to the monograph by Kaplan and Pikelner (1979) for information.

Strangely enough, the authors of theoretical works automatically accept turbulent physics to explain a particular shape of the observed power-law emission spectra for the interstellar gas. To check the possible spectral characteristics of a medium composed of separate objects (clouds) randomly distributed in space with given parameters, we developed an appropriate computer code that simulated the drift curves of the observed medium whose angular spectrum could be obtained by using the same processing software that we applied to the real observations. It immediately emerged that, because of the large extent of the gas layer along the line of sight, the computed angular spectra proved to be close to power-law spectra even if the diameters, densities, and temperatures of the observed objects were fixed. If, however, we use 


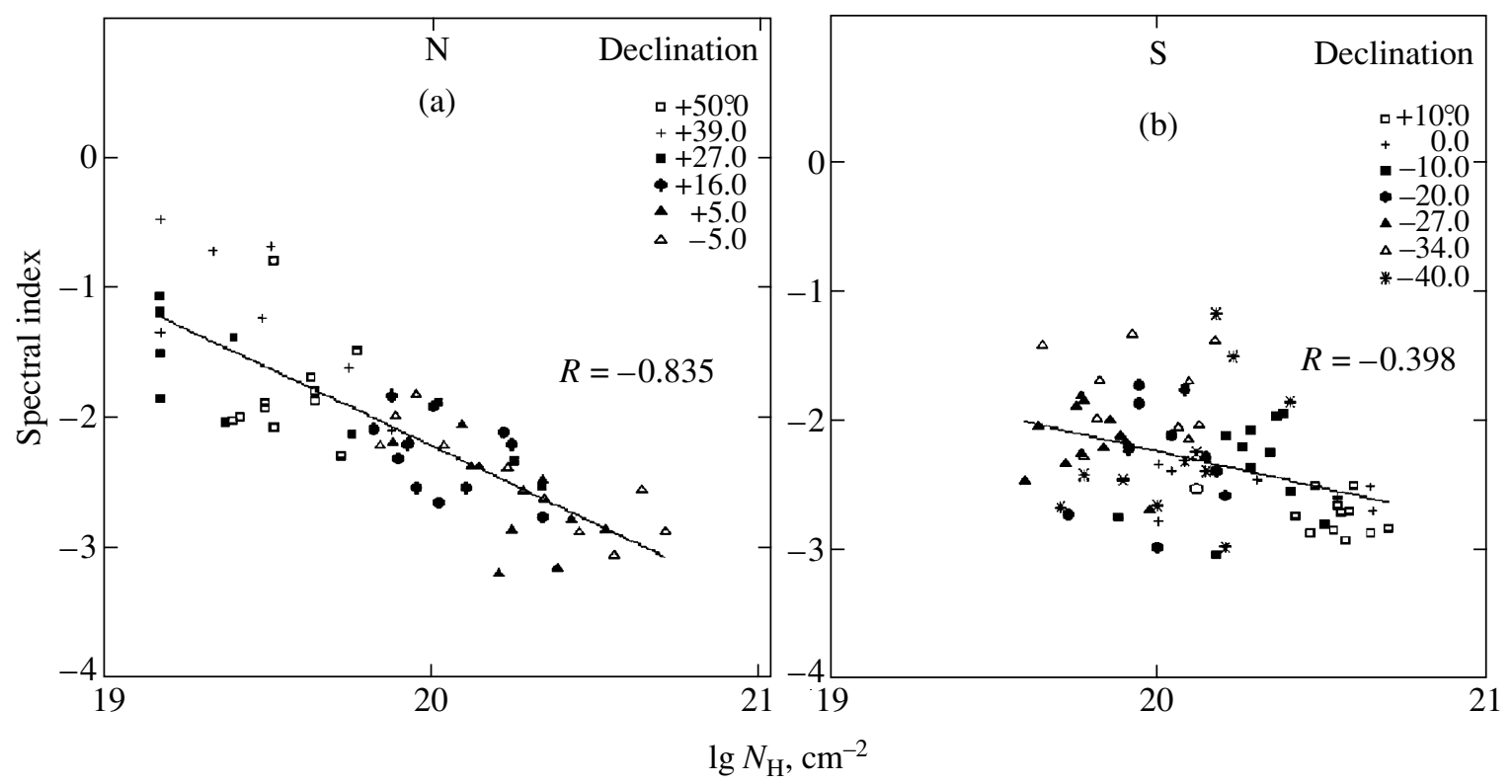

Fig. 5. Indices of the angular spectra versus total H I column density.

the statistical relations for these parameters derived from observations (see, e.g., Gosachinskij and Morozova 1996, 1999), then we can obtain a wide set of indices for the observed angular fluctuation spectrum by choosing appropriate parameters of these relations. Since the number of free parameters in the system of equations was found to be large, choosing the best model takes much time. The results of these computations will be presented in the next paper of this series.

\section{ACKNOWLEDGMENTS}

We wish to thank Z.A. Alferova and T.M. Monastyreva from the Special Astrophysical Observatory (Russian Academy of Sciences) for help with the observations and data reduction. This work was supported by the Russian Foundation for Basic Research (project no. 01-02-17154).

\section{REFERENCES}

1. Z. A. Alferova, I. V. Gosachinskij, S. R. Zhelenkov, and A. S. Morozov, Izv. SAO RAN 23, 89 (1986).

2. J. Ballesteros-Paredes, E. Vasquez-Semadeni, and J. Scalo, Astrophys. J. 515, 286 (1999).

3. J. Crovisier and J. M. Dickey, Astron. Astrophys. 122, 282 (1983).

4. J. M. Dickey, N. M. McClure-Griffiths, S. Stanimirivic, et al., Astrophys. J. 561, 264 (2001).

5. I. Fejes and P. R. Wesselius, Astron. Astrophys. 24, 1 (1973).

6. I. Goldman, Astrophys. J. 541, 701 (2000).

7. I. V. Gosachinskij and V. V. Morozova, Astron. Astrophys. Trans. 11, 215 (1996).

8. I. V. Gosachinskij and V. V. Morozova, Astron. Zh. 76, 883 (1999) [Astron. Rep. 43, 777 (1999)].
9. I. V. Gosachinskij, G. N. Il'in, A. S. Morozov, and V. A. Prozorov, Pis'ma Astron. Zh. 25, 820 (1999) [Astron. Lett. 25, 712 (1999)].

10. D. A. Green, Mon. Not. R. Astron. Soc. 262, 327 (1993).

11. D. Hartmann, P. M. W. Kalberla, W. B. Burton, and U. Mebold, Astron. Astrophys. Suppl. 119, 115 (1996).

12. C. Heiles, S. Kulkarni, and A. A. Stark, Astrophys. J. Lett. 247, L73 (1981).

13. G. N. Il'in, V. A. Prozorov, A. M. Pilipenko, Proceedings of the XXVII Radioastronomy Conference: Problems of Modern Radioastronomy (IPA RAN, St. Petersburg, 1997), p. 128 [in Russian].

14. S. A. Kaplan and S. B. Pikelner, Physics of the Interstellar Medium (Nauka, Moscow, 1979) [in Russian].

15. A. Lazarian, Astron. Astrophys. 293, 507 (1995).

16. A. Lazarian and D. Pogosyan, Astrophys. J. 537, 720 (2000).

17. R. X. McGee and L. M. Newton, Publ. Astron. Soc. Australia 6, 358 (1986).

18. A. P. Venger, I. V. Gosachinskij, V. G. Grachev, and N. F. Ryzhkov, Izv. SAO RAN 14, 118 (1981).

19. A. P. Venger, V. G. Grachev, T. M. Egorova, et al., Soobshch. SAO RAN, №35, 5 (1982).

20. A. P. Venger, I. V. Gosachinskij, N. A. Esepkina, et al., Proceedings of the XXVII Radioastronomy Conference: Problems of Modern Radioastronomy (IPA RAN, St. Petersburg, 1997), p. 130 [in Russian].

Translated by V. Astakhov 